\title{
Y-LineageTracker: a high-throughput analysis framework for Y-chromosomal next-generation sequencing data
}

\author{
Hao Chen ${ }^{1 \dagger}$, Yan Lu ${ }^{1,2 \dagger}$, Dongsheng $L u^{1}$ and Shuhua $X u^{1,3,4,5,6^{*}}$ (D)
}

\author{
${ }^{*}$ Correspondence: \\ xushua@picb.ac.cn \\ ${ }^{\dagger}$ Hao Chen and Yan Lu have \\ contributed equally to this \\ work \\ ${ }^{1}$ Key Laboratory \\ of Computational \\ Biology, Shanghai \\ Institute of Nutrition \\ and Health, University \\ of Chinese Academy \\ of Sciences, Chinese \\ Academy of Sciences, \\ Shanghai 200031, China \\ Full list of author information \\ is available at the end of the \\ article
}

\begin{abstract}
Background: Y-chromosome DNA (Y-DNA) has been used for tracing paternal lineages and offers a clear path from an individual to a known, or likely, direct paternal ancestor. The advance of next-generation sequencing (NGS) technologies increasingly improves the resolution of the non-recombining region of the Y-chromosome (NRY). However, a lack of suitable computer tools prevents the use of NGS data from the Y-DNA studies.
\end{abstract}

Results: We developed Y-LineageTracker, a high-throughput analysis framework that not only utilizes state-of-the-art methodologies to automatically determine NRY haplogroups and identify microsatellite variants of Y-chromosome on a fine scale, but also optimizes comprehensive Y-DNA analysis methods for NGS data. Notably, Y-LineageTracker integrates the NRY haplogroup and Y-STR analysis modules with recognized strategies to robustly suggest an interpretation for paternal genetics and evolution. NRY haplogroup module mainly covers haplogroup classification, clustering analysis, phylogeny construction, and divergence time estimation of NRY haplogroups, and Y-STR module mainly includes Y-STR genotyping, statistical calculation, network analysis, and estimation of time to the most recent common ancestor (TMRCA) based on Y-STR haplotypes. Performance comparison indicated that Y-LineageTracker outperformed existing Y-DNA analysis tools for the high performance and satisfactory visualization effect.

Conclusions: Y-LineageTracker is an open-source and user-friendly command-line tool that provide multiple functions to efficiently analyze Y-DNA from NGS data at both Y-SNP and Y-STR level. Additionally, Y-LineageTracker supports various formats of input data and produces high-quality figures suitable for publication. Y-LineageTracker is coded with Python3 and supports Windows, Linux, and macOS platforms, and can be installed manually or via the Python Package Index (PyPI). The source code, examples, and manual of Y-LineageTracker are freely available at https://www.picb.ac.cn/PGG/ resource.php or CodeOcean (https://codeocean.com/capsule/7424381/tree).

Keywords: Y-chromosome DNA, NGS, NRY haplogroup, Y-STR, Population genetics author(s) and the source, provide a link to the Creative Commons licence, and indicate if changes were made. The images or other third party material in this article are included in the article's Creative Commons licence, unless indicated otherwise in a credit line to the material. If material is not included in the article's Creative Commons licence and your intended use is not permitted by statutory regulation or exceeds the permitted use, you will need to obtain permission directly from the copyright holder. To view a copy of this licence, visit http:// creativecommons.org/licenses/by/4.0/. The Creative Commons Public Domain Dedication waiver (http://creativecommons.org/publi cdomain/zero/1.0/) applies to the data made available in this article, unless otherwise stated in a credit line to the data. 


\section{Introduction}

The human Y-chromosome plays a crucial role in understanding human evolution and genetics [1]. The NRY is one of the most informative regions of the human genome, making it an effective instrument for the study of paternal inheritance [2]. The singlenucleotide polymorphisms (SNPs) and short tandem repeats (STRs) on the NRY have been used as significant markers to trace direct paternal ancestral lineages and reflect the peculiarities of historical male behaviors [3]. In particular, with the increasing popularity of NGS platforms in recent years, the NRY has provided researchers increasingly informative markers to track human paternal lineages [4].

The basic analysis for human paternal lineage study is to infer NRY haplogroups, which were mainly defined by a set of specific Y-SNPs. On the one hand, with the accumulation of Y-chromosome NGS data, several tools have been developed to meet such a need: AMY-tree [5], clean-tree [6], Yleaf [7], and HaploGrouper [8]. These tools support the function of NRY haplogroup inference, but the lack of subsequent genetic analysis based on NRY haplogroup results prevents further tracing and understanding of the patrilineality. On the other hand, with the continuous updating of Y-SNP markers and the topology of the human Y-chromosome tree, such as ISOGG Y-DNA tree (https://www.isogg.org/tree) and YFull tree (https://www.yfull.com/tree), NRY haplogroups classified by some tools were rendered outdated by the latest version. Indeed, it is essential to keep updating the Y-SNP markers and NRY haplogroup nominations up to date for paternal lineage studies. Y-STR polymorphisms provide more informative haplotypes than Y-SNPs do, and they have been widely used in forensics and population genetics $[3,9,10]$. However, at present, the acquisition of Y-STR data relies mainly on experimentation based on polymerase chain reaction (PCR) amplification, and most of the current analyses do not utilize Y-STRs from the high-throughput Y-chromosome sequencing data [11]. With the advance of NGS technologies, various tools have been developed for genotyping STRs from NGS data in recent years. These tools were designed mainly for clinically applications such as TRhist [12], STR-FM [13] and Dante [14], forensic applications such as STRait Razor $[15,16]$, or trait-association studies such as lobSTR [17], HipSTR [18] and popSTR $[19,20]$. Under these circumstances, however, there is a lack of comparable efforts for population genetics and evolutionary studies. In particular, a specific tool or pipeline is required to efficiently make full use of Y-chromosome sequencing data and provide comprehensive analyses with the combination of Y-SNPs and Y-STRs to infer paternal lineage and history in human populations.

To facilitate the Y-DNA data analyses in the context of evolutionary and medical studies, we developed Y-LineageTracker, an open-source and user-friendly tool that standardizes the Y-DNA analysis workflow. Y-LineageTracker assembles a collection of functions applied for human Y-DNA analyses (Tables 1 and 2) and supports multiple inputs and output formats that can communicate with commonly used tools for human genetic analyses such as PAML [21], MEGA [22], and Network (https://www. fluxus-engineering.com), providing a framework to fully analyze human Y-chromosome sequencing data from both Y-SNP and Y-STR levels. The results of the evaluation indicated that Y-LineageTracker can perform faster and more comprehensively with higher resolution and better compatibility than other methods. 
Table 1 Functions implemented in Y-LineageTracker

\begin{tabular}{lll}
\hline Function & Command & Description \\
\hline NRY haplogroup classification & Classify & Classify NRY haplogroups from BAM or VCF file \\
Clustering analysis & Cluster & Perform clustering analysis for NRY haplogroups \\
Phylogeny analysis & Phylo & Perform phylogeny analysis for NRY haplogroups \\
Y-STR genotyping & Genostr & Genotype Y-STRs from BAM or VCF indels \\
Network analysis & Net & Perform network analysis for Y-STR haplotypes \\
Statistical analysis & Stat & Perform statistical analysis for Y-STR haplotypes \\
Time estimation & Time & Estimate NRY haplogroup divergence time \\
& Tmrca & Estimate TMRCA of Y-STR haplotypes \\
\hline
\end{tabular}

Y-DNA analysis functions implemented in Y-LineageTracker. Each function is corresponding to a command

Table 2 Statistical methods applied in Y-LineageTracker

\begin{tabular}{lll}
\hline Function & Method or Statistics & Reference \\
\hline Clustering Analysis & Principal component analysis (PCA) & {$[23]$} \\
& Multidimensional scaling (MDS) & {$[24,25]$} \\
Phylogeny Analysis & Maximum parsimony (MP) & {$[26,27]$} \\
& Identity by state (IBS) & {$[28,29]$} \\
Network Analysis & Median-joining (MJ) & {$[30]$} \\
Statistical Analysis & Haplotype diversity (HD) & {$[31]$} \\
& Mean pairwise distance (MPD) & {$[32]$} \\
& Fixation index (Fst) & {$[33]$} \\
& Genetic diversity (Gst) & {$[34,35]$} \\
& Analysis of molecular variance (AMOVA) & {$[36]$} \\
Time Estimation & Haplogroup prediction from Y-STRs & {$[37]$} \\
& Mcmctree in PAML & {$[21]$} \\
& Rho statistics & {$[38]$}
\end{tabular}

The published Y-DNA analysis methods applied in Y-LineageTracker

\section{Implementation}

Y-LineageTracker is a command-line tool implemented in Python3 with popular NGS data processing and scientific computational Python packages. Y-LineageTracker makes use of Y-chromosome sequencing data and enables subsequent analyses for NRY haplogroups and Y-STRs. As shown in the workflow and main functions of Y-LineageTracker (Fig. 1), Y-LineageTracker contains two analysis modules: NRY haplogroup module and Y-STR module. As for NRY haplogroup module, Y-LineageTracker takes VCF or BAM files as input data for NRY haplogroup classification. The non-sequencing genotype data in VCF are also supported. Y-LineageTracker matches all possible mutations based on the ISOGG Y-DNA tree (2019 version) and determines an optimal track from the terminal haplogroup to Y-Adam, the ancestral Y-chromosome haplotype without any mutation. After NRY haplogroup classification is complete, Y-LineageTracker further 1) applies principal component analysis (PCA) or multidimensional scaling (MDS) clustering method at a specific haplogroup level, 2) constructs a rooted bifurcating phylogenetic tree, and 3) estimates divergence time of haplogroups. For Y-STR module, Y-LineageTracker provides a function to genotype Y-STRs from VCF indels or BAM files; multiple Y-STR genotyping panels are supported, including commonly used 


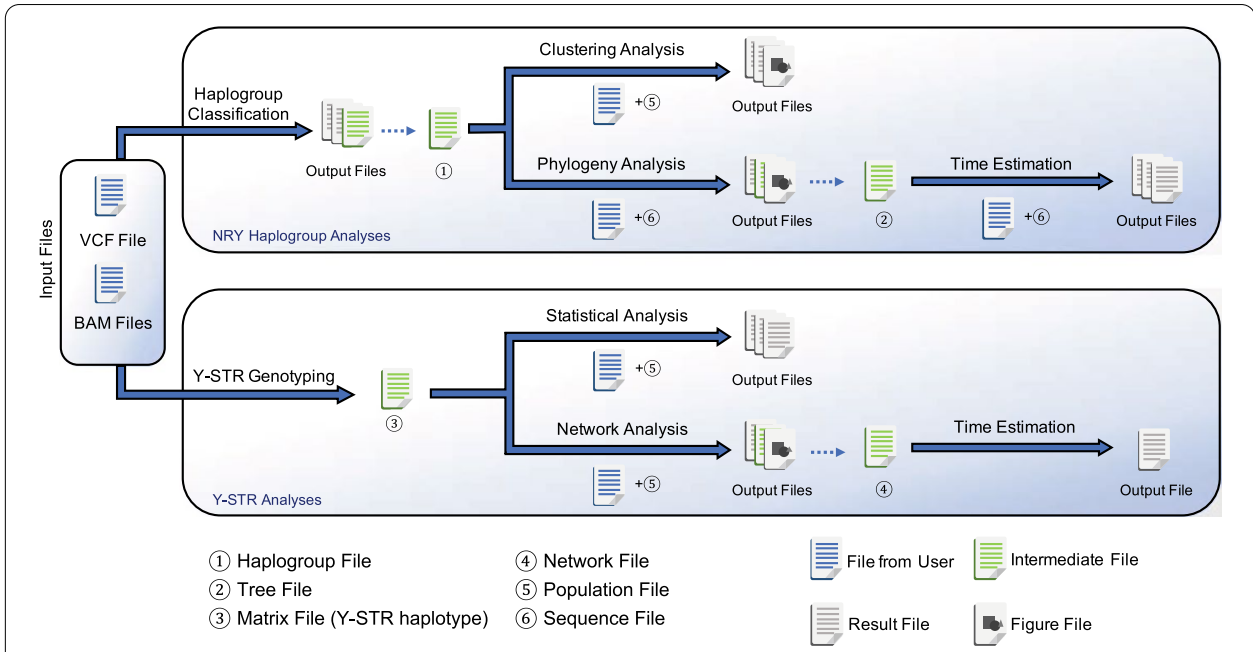

Fig. 1 Workflow and main functions of Y-LineageTracker. The Y-LineageTracker supports taking VCF or BAM files as input data to perform NRY haplogroup classification and Y-STR genotyping. The outputs of haplogroup classification can be used as input data for subsequent clustering and phylogeny analysis, and the outputs of phylogeny analysis can be further used for time estimation. Similarly, the outputs of Y-STR genotyping can be used for statistical analysis and network analysis, and the output of network analysis can be further used for TMRCA estimation

Table 3 Y-STR genotyping panels supported in Y-LineageTracker

\begin{tabular}{|c|c|c|}
\hline Panel & Number of Loci & Description \\
\hline Minimal & 9 & $\begin{array}{l}\text { DYS19, DYS385a, DYS385b, DYS3891, DYS3891I, DYS390, } \\
\text { DYS391, DYS392, DYS393 }\end{array}$ \\
\hline PowerPlex Y & 12 & Minimal + DYS437, DYS438, DYS439 \\
\hline Yfiler & 17 & PowerPlex Y + DYS448, DYS456, DYS458, DYS635 YGATAH4 \\
\hline PowerPlex Y23 & 23 & Yfiler + DYS481, DYS533, DYS549, DYS570, DYS576, DYS643 \\
\hline Named & 92 & All the possible Named Y-STR loci on the Y-chromosome \\
\hline All & 2,451 & All the possible Y-STR loci on the Y-chromosome \\
\hline
\end{tabular}

Multiple Y-STR genotyping panels are supported in Y-LineageTracker and can be used for Y-STR genotyping. The Y-STR genotyping panels refers to YHRD (https://yhrd.org) [41], STRBase (https://strbase.nist.gov) [42] and lobSTR (http://lobst r.teamerlich.org) [17]

commercial Y-STR genotyping kits (Table 3 and Additional file 1). Because comparing genetic differences among populations is a general method of directly unraveling population history, common population statistical analyses for Y-STR haplotypes are included in Y-LineageTracker, such as haplotype diversity, mean pairwise distance, different types of genetic distance, and analysis of molecular variance (AMOVA). Y-LineageTracker also provides a Y-STR haplotype reference data set to infer the most likely NRY haplogroup using the Bayesian approach [37]. Also, users can perform median-joining network analysis and generate a haplotype network plot or a fdi file, which can be directly used as input to draw a network plot or estimate time in the Network software (https://www. fluxus-engineering.com). 


\section{NRY haplogroup classification with tracking lineage}

Before starting NRY haplogroup classification, we firstly divide markers of each haplogroup on the ISOGG Y-DNA tree into two classes: key and ordinary. The key markers are those traditionally used or tested in labs, which are displayed in bold on the ISOGG Y-DNA tree.

To classify haplogroup accurately, Y-LineageTracker first collects all the haplogroups of which most markers are derived alleles. Then, the program selects haplogroups with key mutation marker as key haplogroups and trace possible lineages from the Y-Adam to different downstream and determine the confident terminal haplogroup as the classification result for each sample. When tracing lineages, the program has two criteria: 1) calculating the matching rate of each possible downstream haplogroup and select those are confident; 2) calculating the tracking rate based on the resolution of terminal haplogroup and the number of matched haplogroups in this track. Consider $t$ is the tracking rate for each lineage track:

$$
\begin{aligned}
& t=\frac{\sum_{i=1}^{r} R_{i}}{r} \\
& R= \begin{cases}1 & \left(\text { if } \frac{n}{m} \geq U\right) \\
0 & \text { if } \left.\frac{n}{m}<U\right)\end{cases}
\end{aligned}
$$

where $r$ is the resolution of the terminal haplogroup in each track, $n$ is the number of sites matched to mutations of the haplogroup, $m$ is the number of all the possible mutations for this haplogroup, and $U$ is a cutoff value, the matching rate of haplogroups greater than this value will be selected. As a result, haplogroups with a relatively higher matching rate will be traced, while haplogroups with a lower matching rate but matched to derived allele due to mismatches or sequencing error will be excluded. Next, consider the optimal lineage track that includes haplogroups from the terminal one to the main trunk as $T_{f}$ :

$$
T_{f}=T_{\max \left(t_{1}, t_{2}, \ldots, t_{s}\right)}
$$

where $s$ represents the number of possible tracks, thus track with the most confident tracking rate will be determined. As a result, the final lineage track is a coherent chain with confident haplogroups and a maximum tracking rate.

Finally, the program can also try to detect potential final terminal haplogroup compared to the current terminal key one from its downstream haplogroups without key mutation markers. Samples whose final terminal is not equal to key haplogroup will be annotated in the mutation column of the output file.

For NGS data in VCF, the program also considers the alleles of which all samples are the same to the reference genome and thus not shown in the VCF file. If there are no key haplogroups matched due to the input data is non-sequencing data, or the resolution is relatively low, the program can also apply a similar approach to matched ordinary haplogroups and perform classification analysis. The details of the haplogroup simplification method is provided in Additional file 2. Besides, we also provide the option -ref, which allows users to customize the NRY marker panel in classification module, although we 
attempt to keep updating NRY haplogroup maker panel following the latest version of the ISOGG Y-DNA tree.

\section{Clustering analysis based on NRY haplogroups}

Y-LineageTracker provides clustering analysis to show population structure at the population level. The program takes haplogroup file and population file as input files, then calculates the haplogroup frequency of each population. Based on the haplogroup frequency data, the program calculates eigenvectors and eigenvalues using the PCA method or calculates population pairwise $\mathrm{F}_{\mathrm{ST}}$ then computes positions in the embedding space using the MDS method. For example, to identify population structure using PCA, we can start clustering analysis by a very simple command: LineageTracker cluster -hg file.hg - p file.pop - method pca. After the running is finished, the program outputs the results of the calculation and a scatter plot to show the relationship among populations.

Because the resolution of NRY haplogroups may be different among samples, users can also specify the level of haplogroup resolution using -level option to simplify haplogroups at the relatively same resolution by different simplification methods. For example, command LineageTracker cluster -hg file.hg - p file.pop -method pca -level 3 simplifies haplogroups at level 3. More details of simplification methods in clustering analysis are provided in Additional file 2.

\section{Phylogeny construction based on NRY haplogroups}

Y-LineageTracker provides a function to construct a phylogenetic tree from NRY haplogroups. To construct an NRY phylogenetic tree more confidently and quickly, we here propose a two-step strategy: First, Y-LineageTracker constructs a preliminary tree using haplogroup classification results as a priori inputs, and then it modifies it with multiple sequence alignment data to produce a confident bifurcating tree. The program reads the input NRY haplogroups and converts input haplogroup data to tree structure by assigning each haplogroup to the corresponding position of the ISOGG Y-DNA tree (2019 version). Next, the redundant nodes of the tree will be pruned and modified. However, some nodes of the tree may be polytomies (nodes with more than two children) because some samples have the same haplogroup. Thus, we recommended users to input sequence alignment data as well to construct a bifurcating tree. For example, command LineageTracker phylo-hg file.hg-seq file.fasta-seq-format fasta uses the information of NRY haplogroup and sequence to accurately construct a bifurcating NRY phylogenetic tree. Y-LineageTracker applies tree construction methods such as the unweighted pair group method with arithmetic mean (UPGMA), maximum parsimony and identity-bystate, to generate bifurcating sub-trees in post order (from leaves to root). A sub-tree is a portion of the tree data, the updated sub-tree will be used to replace the polytomy while keeping the original tree structure unchanged. Users can change the tree construction method by the -method option. For example, command LineageTracker phylo - hg file.hg -seq file.fasta-seq-format fasta-method mp applies a method of maximum parsimony to construct the NRY phylogenetic tree. 


\section{Estimation for haplogroup divergence time}

PAML mcmctree [21] is wrapped in Y-LineageTracker to apply the Bayesian MCMC algorithm for estimating haplogroup divergence time. To execute PAML mcmctree program, Y-LineageTracker firstly generates a control file from arguments and then takes required input files (a sequence alignment file, a tree file, and a control file) to perform Bayesian estimation. The tree file should be a rooted bifurcating tree without branch length in newick format. To run PAML mcmctree, fossil calibration is also required. Y-LineageTracker provides commonly used calibration time of haplogroups of Y-DNA tree main trunks to automatically use built-in calibration information based on the input tree. The outputs of time estimation include raw outputs of the mcmctree and results summarized from them, which gives the estimated time of each tree node.

\section{Y-STR genotyping}

To genotype Y-STR loci from Y-chromosome sequencing data, users just need to provide input file and the version of reference genome as arguments and type a very simple command: LineageTracker genostr - bam file.bam - b 38. Y-LineageTracker also provides a different genotyping panel for Y-STR genotyping (Table 3). The Y-STR genotyping panel of hg19 refers to YHRD [41], STRBase [42] and lobSTR [17], then we apply LiftOver software (https://genome.sph.umich.edu/wiki/LiftOver) to get reference panel of hg38 as well. Unnamed Y-STRs with repeat numbers less than four or irregular motif arrangements were dropped.

As shown in the flow chart (Fig. 2), to genotype a specific Y-STR locus, Y-LineageTracker firstly gets the reference Y-STR locus from the reference panel. Consider the repeat sequence of the reference Y-STR locus is $L$, which can be represented as: $L=\left(M_{1}\right)_{p_{1}}\left(M_{2}\right)_{p_{2}} \cdots\left(M_{n}\right)_{p_{n}}$, where $M_{1}, M_{2} \cdots M_{n}$ are motifs of the Y-STR locus, $p_{i}$ represents the repeat number of the $\mathrm{i}^{\text {th }}$ motif. Thus, the sum of repeat number of this Y-STR locus is: $p=\sum_{i=1}^{n} p_{i}$.

Based on this sequence information, let the initial sequence $R$ of the Y-STR locus of one sample be forward extended from $L: R=L^{\prime}(N)_{s}$, where $L^{\prime}$ is the sequence at the same position of the reference and $L^{\prime}=\left(M_{1}\right)_{q_{1}}\left(M_{2}\right)_{q_{2}} \cdots\left(M_{n}\right)_{q_{n}}, s$ represents the motif length of the Y-STR locus, $(N)_{s}$ represents the sequence with equal motif length behind this Y-STR locus and $q_{i}$ represents the repeat number of the $\mathrm{i}^{\text {th }}$ motif on initial sequence $R$. As a result, the total repeat number of this extended sequence is: $q=\sum_{i=1}^{n} q_{i}$. Finally, the repeat number of $R$ can be compared with the reference $L: 1)$ if the $q$ is equal to or less than $p$, the repeat number is $q, 2)$ if the $q$ is equal to $p+1$, extend the sequence in the same way again and replace $p$ to $p+1$ and update $q$ of the extended sequence, and repeat this step until the $p$ is equal to updated $q$, which means there are no more motifs extended from the sequence.

\section{Results and discussion}

\section{Example outputs}

For NRY haplogroup module, analysis workflow starts from the NRY haplogroup classification. Additional file 3 gives an example of NRY haplogroup classification 


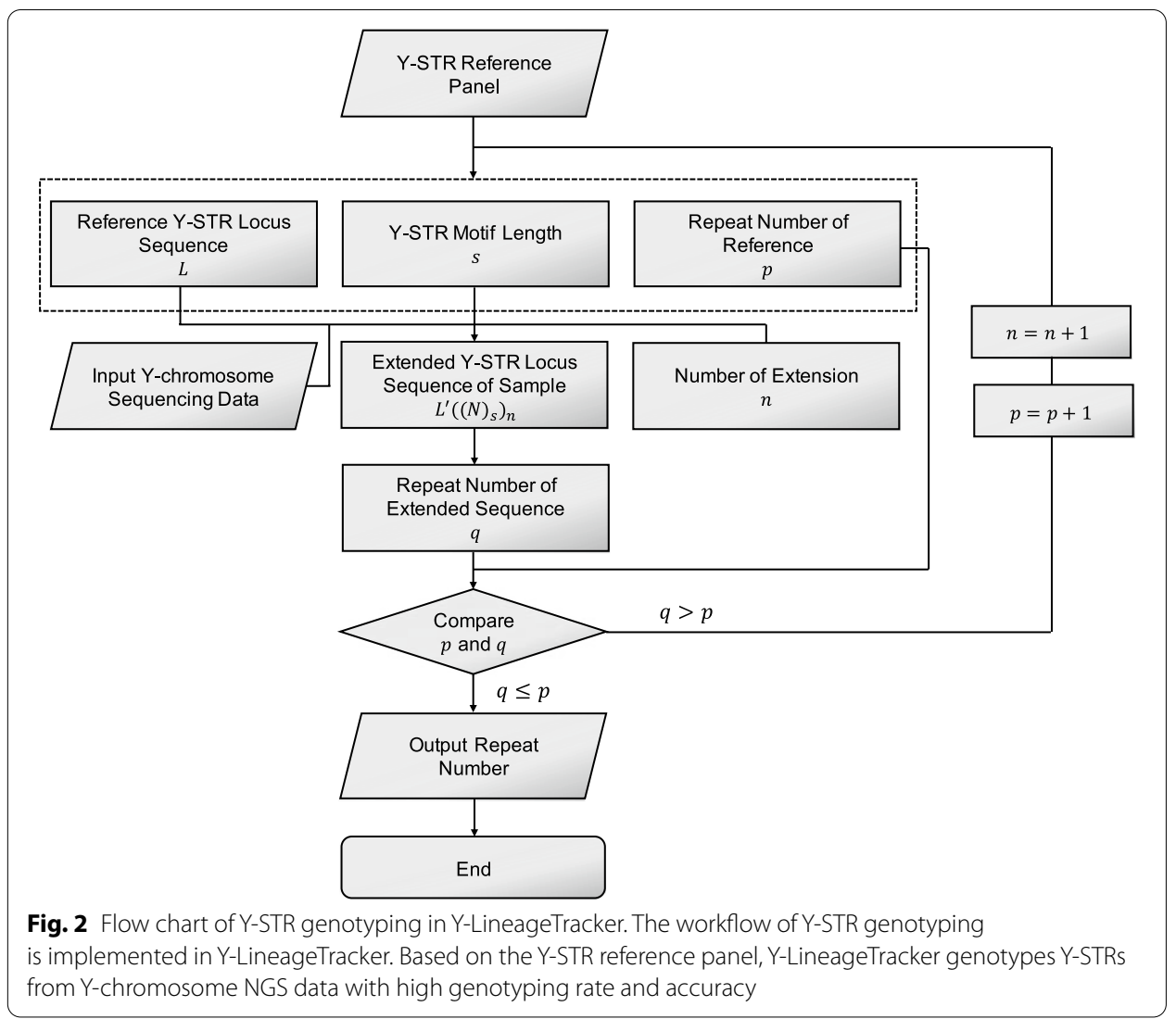

results of 1,233 male samples from the 1000 Genomes Project [43]. The haplogroup classification results provided by Y-LineageTracker, were compared and validated with the published NRY haplogroup results of the 1000 Genomes Project [1]. We reassigned the terminal mutation makers of the published haplogroup results to the latest ISOGG Y-DNA tree (2019 version) as the haplogroups to be compared with Y-LineageTracker. The results indicated that the NRY haplogroups classified by Y-LineageTracker are consistent with the published results with relatively higher resolution (Additional file 4). In other words, Y-LineageTracker provides sufficient performance to output reliable haplogroup classification results, which is important for subsequent analyses based on NRY haplogroups. Further, based on the haplogroup results, we applied PCA and MDS of clustering analysis function in Y-LineageTracker to directly produce figures that display the population structure of the studied samples (Fig. 3a, b). We also performed phylogenetic analysis in Y-LineageTracker to construct an NRY phylogenetic tree using haplogroup classification results of male samples of Han Chinese in Beijing (CHB), Southern Han Chinese (CHS), and Utah Residents with Northern and Western European ancestry (CEU) from the 1000 Genomes Project (Fig. 3c).

In order to analyze populations of close relationship to a fine-scale, Y-STR analysis within the same NRY haplogroup is recommended. We selected Chinese samples (CHB, CHS, and CDX) with NRY haplogroup O2 and genotyped Y-STRs under the minimal Y-STR genotyping panel. We next applied the network analysis function to do network analysis based on the Y-STR haplotypes (Fig. 4). The result showed that the nodes in the 


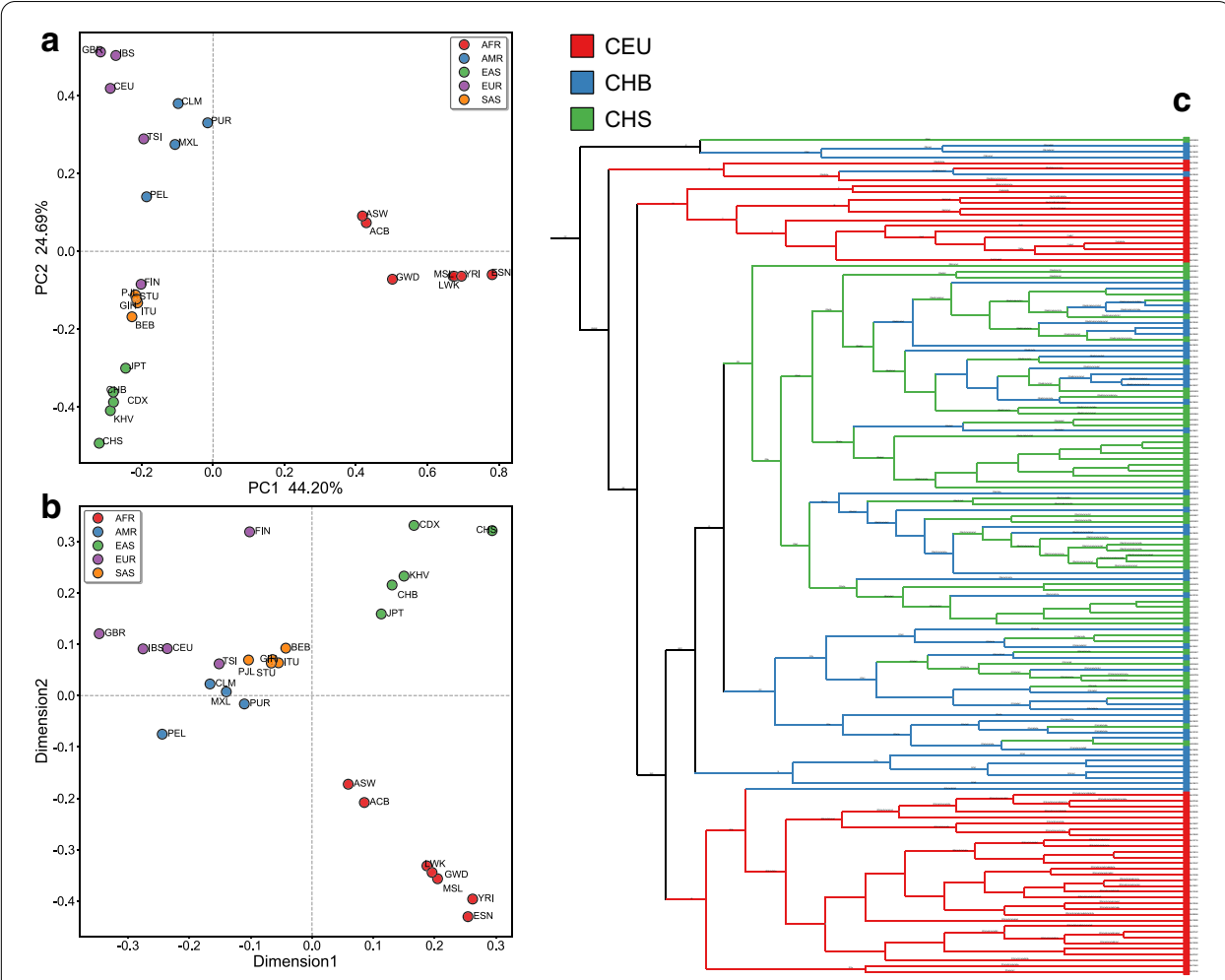

Fig. 3 Example output figures of NRY haplogroup analysis performed by Y-LineageTracker. a Principal component analysis (PCA) and $\mathbf{b}$ multidimensional scaling (MDS) performed by Y-LineageTracker respectively, based on NRY haplogroups of 1, 233 male samples from the 1000 Genomes Project data set phase 3. c Phylogeny analysis performed by Y-LineageTracker, based on NRY haplogroups of 147 male samples from the 1000 Genomes Project CEU, CHB, and CHS

network are admixed, which indicates that there is no significant difference in Y-STR holotypes among three Chinese populations under the haplogroup O2.

\section{Performance comparison}

To evaluate the Y-LineageTracker performance, we compared Y-LineageTracker to other published NRY haplogroup classification tools in different ways. We referred to published NRY haplogroup results of samples from the 1000 Genomes Project [1] and used sequencing data of male samples from the 1000 Genomes Project [43] as input data to run different tools and compare the classification results and time costs. Results showed that Y-LineageTracker performs better with respect to data compatibility for supporting NGS data in VCF or BAM format and can take multiple files as input data to start NRY haplogroup classification analysis (Table 4). The comparison of haplogroup classification results confirmed that (1) when the input file is VCF, Y-LineageTracker classifies NRY haplogroups with higher resolution than the AMY-Tree and with sufficient resolution as good as the HaploGrouper (Fig. 5a and Additional file 4), (2) when input files are BAMs, the resolution of haplogroup classification results is higher than that of the clean-tree and is as high as that of the Yleaf (Fig. 5b and Additional file 5). In addition, the runtime comparison showed that no matter whether the format of input data is BAM or VCF, Y-LineageTracker performed faster in haplogroup classification than other published 


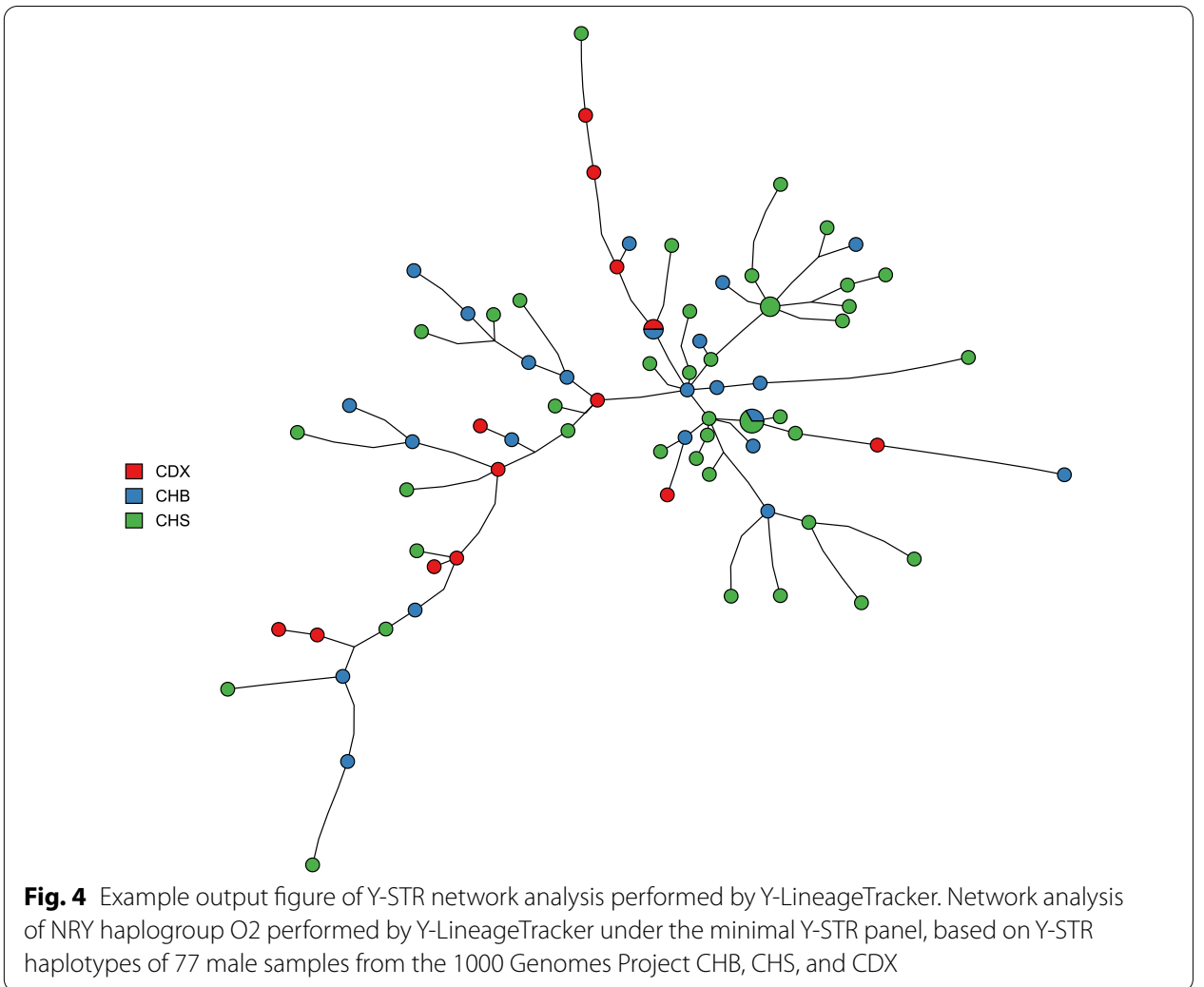

Table 4 General comparison of NRY haplogroup classification tools

\begin{tabular}{|c|c|c|c|c|c|c|}
\hline Tool & Version & $\begin{array}{l}\text { Programming } \\
\text { Language }\end{array}$ & $\begin{array}{l}\text { Supported Input } \\
\text { Format }\end{array}$ & $\begin{array}{l}\text { Sample Input } \\
\text { form }\end{array}$ & $\begin{array}{l}\text { Number of } \\
\text { Markers }\end{array}$ & $\begin{array}{l}\text { Supported } \\
\text { Reference }\end{array}$ \\
\hline Y-LineageTracker & 1.3.0 & Python & VCF, BAM/CRAM & Multiple & 74,570 & hg19, hg38 \\
\hline HaploGrouper & 1.0 & Python & VCF & Multiple & 71,406 & hg19, hg38 \\
\hline AMY-Tree & 2.0 & Perl & VCF-like & Single & 5,925 & hg18, hg19 \\
\hline Yleaf & 2.2 & Python & $\begin{array}{l}\text { FASTQ, BAM/ } \\
\text { CRAM }\end{array}$ & Single & 65,459 & hg19, hg38 \\
\hline clean-tree & 2.0 & Python, R & BAM & Single & 539 & hg19 \\
\hline
\end{tabular}

General comparison of currently available NRY haplogroup classification tools. The sample input form means whether the tool can take multiple files or a single file as input to perform analysis

tools (Fig. 6). Our implementation not only provides high-resolution haplogroup classification results but also reduces computational time.

We also compared Y-STR genotyping results with lobSTR [17] under the Y-STR minimal genotyping panel. The results show that the repeat numbers of Y-STR loci genotyped from Y-LineageTracker are almost consistent with the results of lobSTR, and the Y-STR genotyping rate is higher than that of lobSTR (Additional file 6). Our implementation is not only sufficient to genotype Y-STR loci in specific panels but also provide a higher genotyping rate. 

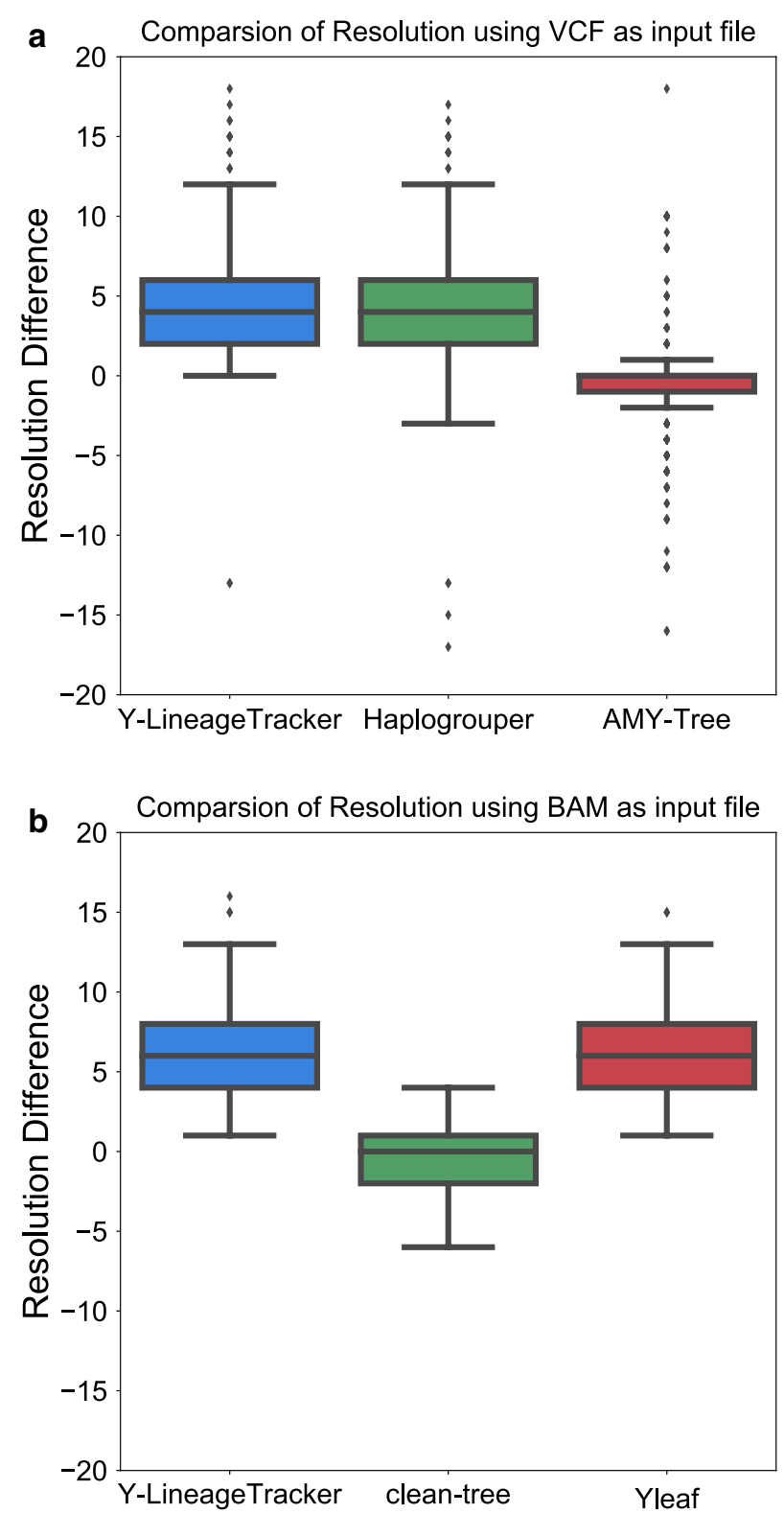

Fig. 5 Resolution comparison of NRY haplogroup classification tools. Resolution differences of NRY haplogroup classification tools that use $\mathbf{a}$ VCF and $\mathbf{b}$ BAM as input files respectively, compared with published NRY haplogroup results of the 1000 Genomes Project. The difference value is calculated by the resolution of haplogroups classified by tools minus the resolution of the published haplogroups. Positive values indicate the resolution of haplogroups is higher than those that are published, and negative values indicate the resolution of haplogroups is lower than those that are published

\section{Application scenarios}

We have integrated and improved analysis methods for the NRY with sufficient performance. Our methods provide a commonly used analysis pipeline for the NRY both at Y-SNP and Y-STR levels. We prospect that in the future, with the continuous increase of the public NSG data, Y-LineageTracker can facilitate uncovering population history. For example, combined with analyses of mitochondrial DNA, Y-LineageTracker 

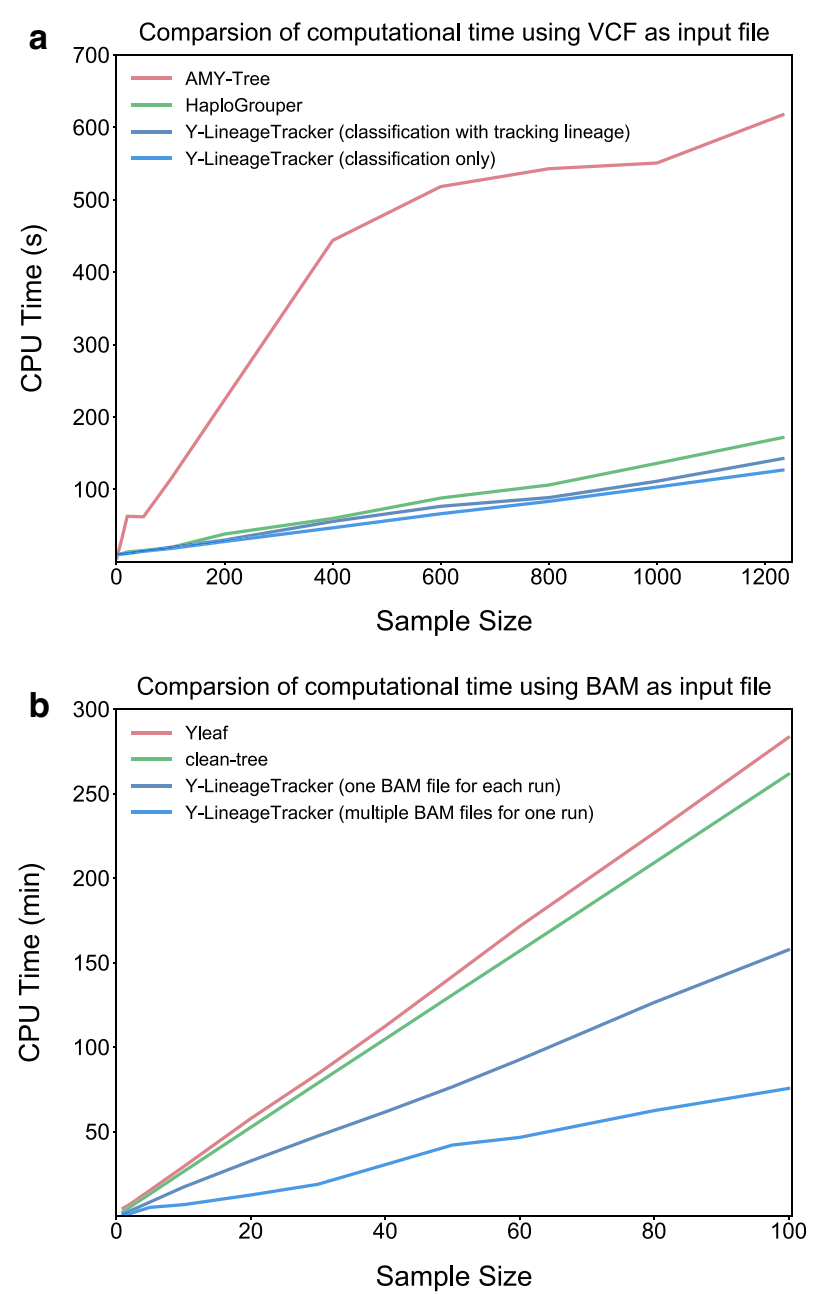

Fig. 6 Time costs of NRY haplogroup classification tools. Comparison of time cost of NRY haplogroup classification tools that use $\mathbf{a}$ VCF and $\mathbf{b}$ BAM as input files, respectively. The evaluation of time costs is performed on the local machine with 64-bit Red Hat 6.3, CPU AMD Opteron (TM) Processor 6234, and 512 GB RAM. We randomly select male samples with different sample sizes from the 1000 Genomes Project data set phase 3 and perform classification analysis using the same data by different tools. The numbers of variants of VCFs are 62,043

demonstrated the potential for the detection of sex-biased demography. We also look forward to combining with data of autosome, X-chromosome, and mitochondrial DNA to develop and optimize analysis methods to reconstruct paternal lineages of human genetics and evolution from multiple perspectives.

\section{Conclusions}

We developed Y-LineageTracker, an effective and flexible tool that can standardize analysis workflow for human Y-chromosome sequencing data while performing at a high level. There are two major function modules in Y-LineageTracker, based on NRY haplogroups and Y-STRs, respectively. Y-LineageTracker can not only classify NRY haplogroups and genotype Y-STRs from NGS data with high resolution but also support subsequent analyses for NRY haplogroups and Y-STR haplotypes. We propose that 
Y-LineageTracker provides an analysis framework to investigate human evolution, population history, and sex-biased demography from Y-chromosome NGS data.

\section{Availability and requirements}

Project name: Y-LineageTracker

Project home page: https://www.picb.ac.cn/PGG/resource.php

Operating system: Platform independent

Programming language: Python3

Other requirements: Python packages (numpy, pandas, scipy, scikit-learn, matplotlib, ete3, pysam, and networkx)

License: MIT

Any restrictions to use by non-academics: none

\section{Abbreviations}

DNA: Deoxyribonucleic acid; NGS: Next-generation sequencing; NRY: Non-recombining region of the Y-chromosome; SNP: Single-nucleotide polymorphism; STR: Short tandem repeat; TMRCA: Time to the most recent common ancestor; PyPI: Python package index; ISOGG: International society of genetic genealogy; PCR: Polymerase chain reaction; VCF: Variant call format; BAM: Binary alignment map; PCA: Principal component analysis; MDS: Multidimensional scaling; AMOVA: Analysis of molecular variance; UPGMA: Unweighted pair group method with arithmetic mean; MCMC: Markov chain Monte Carlo; YHRD: Y-Chromosome STR Haplotype Reference Database; CHB: Han Chinese in Beijing, China; CHS: Southern Han Chinese; CEU: Utah residents with Northern and Western European ancestry; CDX: Chinese Dai in Xishuangbanna, China.

\section{Supplementary Information}

The online version contains supplementary material available at https://doi.org/10.1186/s12859-021-04057-z.

Additional file 1: Table S1. Y-STR reference panel used for Y-STR genotyping. This table gives a detailed reference panel for Y-STR genotyping applied in Y-LineageTracker.

Additional file 2: Text.

Additional file 3: Table S2. Example output of NRY classification results generated by Y-LineageTracker. This table gives an example of the Y-LineageTracker output result of 1, 233 male samples from the 1000 Genomes Project data set phase 3.

Additional file 4: Table S3. Result comparison of NRY haplogroup classification tools on VCF data. This table gives a comparison of NRY haplogroup results of 1, 233 male samples from the 1000 Genomes Project data set phase 3 in VCF, performed by different NRY haplogroup classification tools that can use VCF data as input files, including Y-LineageTracker, HaploGrouper, and AMY-Tree.

Additional file 5: Table S4. Result comparison of NRY haplogroup classification tools on BAM data. This table gives a comparison of NRY haplogroup results of 100 male samples from the 1000 Genomes Project data set phase 3 in BAM format, performed by different NRY haplogroup classification tools that can use BAM data as input files, including Y-LineageTracker, Yleaf, and clean-tree.

Additional file 6: Table S5. Result comparison of Y-STR genotyping tools. This table gives a comparison of Y-STR genotypes of 100 male samples from the 1000 Genomes Project data set phase 3 in BAM format, performed by Y-LineageTracker and lobSTR.

\section{Acknowledgments}

We are grateful to the people of PGG group who participated in the software testing and provided constructive suggestions

\section{Authors' contributions}

SX conceived the study. SX and YL designed and supervised the study. HC and DL wrote the code. HC improved the code and performed data analyses. HC and YL drafted manuscript. SX revised manuscript. All authors read and approved the final manuscript. 


\section{Funding}

This study is supported by the National Key Research and Development Program (2016YFC0906403), the National Natural Science Foundation of China (NSFC) grant (32030020, 32041008, 31525014, 31771388, and 31961130380), the UK Royal Society-Newton Advanced Fellowship (NAF\R1\191094), Key Research Program of Frontier Sciences (QYZDJSSW-SYS009) and the Strategic Priority Research Program (XDB38000000) of the Chinese Academy of Sciences, and the Shanghai Municipal Science and Technology Major Project (2017SHZDZX01).

\section{Availability of data and materials}

Source code is available at https://www.picb.ac.cn/PGG/resource.php. The 1000 Genomes Project data analyzed in the current study are available at https://www.internationalgenome.org/data. Data generated in this study are included in the additional files.

\section{Declarations}

Ethics approval and consent to participate

Not applicable.

\section{Consent for publication}

Not applicable.

\section{Competing interests}

The authors declare no competing interests.

\section{Author details}

${ }^{1}$ Key Laboratory of Computational Biology, Shanghai Institute of Nutrition and Health, University of Chinese Academy of Sciences, Chinese Academy of Sciences, Shanghai 200031, China. ${ }^{2}$ School of Life Sciences, Fudan University, Shanghai 200433, China. ${ }^{3}$ School of Life Science and Technology, ShanghaiTech University, Shanghai 201210, China. ${ }^{4}$ Center for Excellence in Animal Evolution and Genetics, Chinese Academy of Sciences, Kunming 650223, China. ${ }^{5}$ Henan Institute of Medical and Pharmaceutical Sciences, Zhengzhou University, Zhengzhou 450052, China. ${ }^{6}$ Collaborative Innovation Center of Genetics and Development, Fudan University, Shanghai 200438, China.

Received: 2 December 2020 Accepted: 28 February 2021

Published online: 09 March 2021

\section{References}

1. Poznik GD, Xue Y, Mendez FL, Willems TF, Massaia A, Wilson Sayres MA, Ayub Q, McCarthy SA, Narechania A, Kashin S, et al. Punctuated bursts in human male demography inferred from 1,244 worldwide Y-chromosome sequences. Nat Genet. 2016;48(6):593-9.

2. Poznik GD, Henn BM, Yee MC, Sliwerska E, Euskirchen GM, Lin AA, Snyder M, Quintana-Murci L, Kidd JM, Underhill $P A$, et al. Sequencing $Y$ chromosomes resolves discrepancy in time to common ancestor of males versus females. Science. 2013;341(6145):562-5.

3. Jobling MA, Tyler-Smith C. Human Y-chromosome variation in the genome-sequencing era. Nat Rev Genet. 2017;18(8):485-97.

4. Hallast P, Batini C, Zadik D, Maisano Delser P, Wetton JH, Arroyo-Pardo E, Cavalleri GL, de Knijff P, Destro Bisol G, Dupuy BM, et al. The Y-chromosome tree bursts into leaf: 13,000 high-confidence SNPs covering the majority of known clades. Mol Biol Evol. 2015;32(3):661-73.

5. Van Geystelen A, Decorte R, Larmuseau MH. AMY-tree: an algorithm to use whole genome SNP calling for Y chromosomal phylogenetic applications. BMC Genom. 2013;14:101.

6. Ralf A, van Oven M, Zhong K, Kayser M. Simultaneous analysis of hundreds of Y-chromosomal SNPs for high-resolution paternal lineage classification using targeted semiconductor sequencing. Hum Mutat. 2015;36(1):151-9.

7. Ralf A, Montiel Gonzalez D, Zhong K, Kayser M. Yleaf: software for human Y-chromosomal haplogroup inference from next-generation sequencing data. Mol Biol Evol. 2018;35(5):1291-4.

8. Jagadeesan A, Ebenesersdottir SS, Guethmundsdottir VB, Thordardottir EL, Moore KHS, Helgason A. HaploGrouper: a generalized approach to haplogroup classification. Bioinformatics 2020.

9. Jobling MA, Pandya A, Tyler-Smith C. The Y chromosome in forensic analysis and paternity testing. Int J Legal Med. 1997;110(3):118-24

10. Kayser M, de Knijff P, Dieltjes P, Krawczak M, Nagy M, Zerjal T, Pandya A, Tyler-Smith C, Roewer L. Applications of microsatellite-based Y chromosome haplotyping. Electrophoresis. 1997;18(9):1602-7.

11. Willems T, Gymrek M, Poznik GD, Tyler-Smith C, Genomes Project Chromosome YG, Erlich Y. Population-scale sequencing data enable precise estimates of Y-STR mutation rates. Am J Hum Genet. 2016;98(5):919-33.

12. Doi K, Monjo T, Hoang PH, Yoshimura J, Yurino H, Mitsui J, Ishiura H, Takahashi Y, Ichikawa Y, Goto J, et al. Rapid detection of expanded short tandem repeats in personal genomics using hybrid sequencing. Bioinformatics. 2014;30(6):815-22.

13. Fungtammasan A, Ananda G, Hile SE, Su MS, Sun C, Harris R, Medvedev P, Eckert K, Makova KD. Accurate typing of short tandem repeats from genome-wide sequencing data and its applications. Genome Res. 2015;25(5):736-49.

14. Budis J, Kucharik M, Duris F, Gazdarica J, Zrubcova M, Ficek A, Szemes T, Brejova B, Radvanszky J. Dante: genotyping of known complex and expanded short tandem repeats. Bioinformatics. 2019;35(8):1310-7.

15. Warshauer DH, King JL, Budowle B. STRait Razor v.20: the improved STR Allele Identification Tool-Razor. Forensic Sci Int Genet. 2015;14:182-6. 
16. Warshauer DH, Lin D, Hari K, Jain R, Davis C, Larue B, King JL, Budowle B. STRait Razor: a length-based forensic STR allele-calling tool for use with second generation sequencing data. Forensic Sci Int Genet. 2013;7(4):409-17.

17. Gymrek M, Golan D, Rosset S, Erlich Y. lobSTR: A short tandem repeat profiler for personal genomes. Genome Res. 2012;22(6):1154-62.

18. Willems T, Zielinski D, Yuan J, Gordon A, Gymrek M, Erlich Y. Genome-wide profiling of heritable and de novo STR variations. Nat Methods. 2017;14(6):590-2.

19. Kristmundsdottir S, Eggertsson HP, Arnadottir GA, Halldorsson BV. popSTR2 enables clinical and population-scale genotyping of microsatellites. Bioinformatics. 2020;36(7):2269-71.

20. Kristmundsdottir S, Sigurpalsdottir BD, Kehr B, Halldorsson BV. popSTR: population-scale detection of STR variants. Bioinformatics. 2017;33(24):4041-8.

21. Yang Z. PAML 4: phylogenetic analysis by maximum likelihood. Mol Biol Evol. 2007:24(8):1586-91.

22. Kumar S, Stecher G, Li M, Knyaz C, Tamura K. MEGA X: molecular evolutionary genetics analysis across computing platforms. Mol Biol Evol. 2018;35(6):1547-9.

23. Patterson N, Price AL, Reich D. Population structure and eigenanalysis. PLoS Genet. 2006;2(12):e190

24. Tzeng J, Lu HH, Li WH. Multidimensional scaling for large genomic data sets. BMC Bioinform. 2008;9:179.

25. Taguchi YH, Oono Y. Relational patterns of gene expression via non-metric multidimensional scaling analysis. Bioinformatics. 2005;21(6):730-40.

26. Nixon K. The Parsimony Ratchet, a new method for rapid parsimony analysis. Cladistics. 1999;15:407-14

27. Felsenstein J. Confidence limits on phylogenies: an approach using the bootstrap. Evolution. 1985;39(4):783-91.

28. Weir BS, Anderson AD, Hepler AB. Genetic relatedness analysis: modern data and new challenges. Nat Rev Genet. 2006;7(10):771-80.

29. Milligan BG. Maximum-likelihood estimation of relatedness. Genetics. 2003;163(3):1153-67.

30. Bandelt HJ, Forster P, Rohl A. Median-joining networks for inferring intraspecific phylogenies. Mol Biol Evol. 1999;16(1):37-48.

31. Nei M, Tajima F. DNA polymorphism detectable by restriction endonucleases. Genetics. 1981;97(1):145-63.

32. Webb CO, Ackerly DD, McPeek MA, Donoghue MJ. Phylogenies and community ecology. Annu Rev Ecol Syst. 2002;33:475-505.

33. Weir BS, Hill WG. Estimating F-statistics. Annu Rev Genet. 2002;36:721-50.

34. Hedrick PW. A standardized genetic differentiation measure. Evolution. 2005;59(8):1633-8.

35. Nei M. Analysis of gene diversity in subdivided populations. Proc Natl Acad Sci USA. 1973;70(12):3321-3.

36. Excoffier L, Smouse PE, Quattro JM. Analysis of molecular variance inferred from metric distances among DNA haplotypes: application to human mitochondrial DNA restriction data. Genetics. 1992;131(2):479-91.

37. Athey TW. Haplogroup prediction from Y-STR values using a bayesian-allele-frequency approach. J Genet Geneal. 2006;2:34-9.

38. Forster $\mathrm{P}$, Harding R, Torroni A, Bandelt HJ. Origin and evolution of Native American mtDNA variation: a reappraisal. Am J Hum Genet. 1996;59(4):935-45.

39. Sengupta S, Zhivotovsky LA, King R, Mehdi SQ, Edmonds CA, Chow CE, Lin AA, Mitra M, Sil SK, Ramesh A, et al. Polarity and temporality of high-resolution $\mathrm{y}$-chromosome distributions in India identify both indigenous and exogenous expansions and reveal minor genetic influence of Central Asian pastoralists. Am J Hum Genet. 2006;78(2):202-21.

40. Goldstein DB, Ruiz Linares A, Cavalli-Sforza LL, Feldman MW. Genetic absolute dating based on microsatellites and the origin of modern humans. Proc Natl Acad Sci USA. 1995;92(15):6723-7.

41. Willuweit S, Roewer L. The new Y chromosome haplotype reference database. Forensic Sci Int Genet. 2015;15:43-8.

42. Ruitberg CM, Reeder DJ, Butler JM. STRBase: a short tandem repeat DNA database for the human identity testing community. Nucleic Acids Res. 2001;29(1):320-2.

43. The 1000 Genomes Project Consortium. A global reference for human genetic variation. Nature 2015;526(7571):68-74.

\section{Publisher's Note}

Springer Nature remains neutral with regard to jurisdictional claims in published maps and institutional affiliations.

Ready to submit your research? Choose BMC and benefit from:

- fast, convenient online submission

- thorough peer review by experienced researchers in your field

- rapid publication on acceptance

- support for research data, including large and complex data types

- gold Open Access which fosters wider collaboration and increased citations

- maximum visibility for your research: over $100 \mathrm{M}$ website views per year

At BMC, research is always in progress.

Learn more biomedcentral.com/submissions 